\title{
Interferometry of the Compound Nucleus
}

\author{
S. E. Koonin, ${ }^{(1)}$ W. Bauer, ${ }^{(1,2)}$ and A. Schäfer ${ }^{(1)}$ \\ ${ }^{(1)}$ W. K. Kellogg Radiation Laboratory, California Institute of Technology, Pasadena, California 91125 \\ ${ }^{(2)}$ National Superconducting Cyclotron Laboratory (NSCL) and Physics Department, \\ Michigan State University, East Lansing, Michigan 48824 \\ (Received 28 November 1988)
}

\begin{abstract}
We consider interference effects in the inclusive emission of two identical particles from a compound nucleus. Statistical assumptions result in a correlation of the emitted particles that depends upon the coherence properties of the level widths in a form similar to that describing fluctuations in an exclusive cross section. A schematic application to the decay of a rapidly rotating compound nucleus shows that the observed source size can depend upon the kinematics of the measurement.
\end{abstract}

PACS numbers: 25.70.Gh, 24.60.-k

Most aspects of nuclear collisions involving momenta larger than the Fermi momentum of nuclear matter are well described by classical considerations (e.g., Boltzmann-Uehling-Uhlenbeck, cascade, or fluid-dynamic simulations). ${ }^{1}$ However, quantal phenomena can be relevant for those final states in which the relative wavelength of two detected particles is small compared to the size of the system. Analysis of the resulting structure in the two-particle inclusive cross section is generally termed "interferometry" in analogy with the HanburyBrown-Twiss ${ }^{2}$ method for determining stellar sizes, although final-state interactions between the detected particles and the symmetry or antisymmetry of their wave function must be included for a proper analysis.

To date, interferometry has been performed with protons, pions, neutrons, and light nuclei for heavy-ion collisions from 10's of $\mathrm{MeV}$ per nucleon, as well as for nucleon-nucleon and electron-positron collisions. ${ }^{3}$ A typical analysis assumes a source distribution (e.g., Gaussian) for the emitted particles parametrized in terms of a size and lifetime (and occasionally an anisotropy) and proceeds to determine these parameters by fitting the data. Such studies are likely to become even more important as ultrarelativistic heavy-ion beams are exploited. ${ }^{4}$

The interpretation of these parameters is, at best, phenomenological. While plausible "derivations" of the connection between the source distribution and the twoparticle inclusive cross section can be given, ${ }^{5}$ a rigorous foundation is lacking. Any observable reaction must be expressed ultimately in terms of $S$-matrix elements, but we have no way to relate the source distribution to these more fundamental quantities. We also lack the proper procedure for generating interferometric patterns from the simulations currently used to describe nuclear collisions. ${ }^{1}$

In this Letter, we take a step toward addressing some of these questions by considering interferometry within the standard statistical description of the compound nucleus. In particular, we consider an equilibrated com- pound nucleus decaying through the emission of two or more identical particles (nucleons, pions, photons, etc.). We show how the two-particle correlation function (viz., the "size" and "lifetime" of the source) is related to coherence in the decay amplitudes and the average properties of the compound-nuclear states.

We assume that the reaction proceeds from the initial channel $i$ through an equilibrated compound nucleus to an evaporation residue in state $\gamma$ by the sequential emission of identical particles of momenta $\mathbf{p}_{1}$ and $\mathbf{p}_{2}$, with energies $E_{1}$ and $E_{2}$, respectively. The corresponding $T$ matrix element at total energy $E$ is

$$
\begin{aligned}
T_{\gamma i}\left(\mathbf{p}_{2}, \mathbf{p}_{1}\right)=\sum_{\alpha, \beta} \gamma_{\gamma \beta}\left(\mathbf{p}_{2}\right) & \frac{1}{E-\epsilon_{\beta}-i \Gamma_{\beta} / 2-E_{1}} \\
& \times \gamma_{\beta \alpha}\left(\mathbf{p}_{1}\right) \frac{1}{E-\epsilon_{\alpha}-i \Gamma_{\alpha} / 2} \gamma_{\alpha i} .
\end{aligned}
$$

Here, $\alpha$ and $\beta$ are the intermediate compound-nucleus levels, with energies $\epsilon_{\alpha}$ and $\epsilon_{\beta}$ and total widths $\Gamma_{\alpha}$ and $\Gamma_{\beta}$, respectively. $\gamma_{\nu \mu}(\mathbf{p})$ is the reduced width for compoundnucleus state $\mu$ to decay to $v$ by the emission of a particle of momentum $\mathbf{p}$, and $\gamma_{\alpha i}$ is the reduced width for the entrance channel.

The two-particle inclusive cross section is proportional to the square of the coherent sum of the amplitudes corresponding to the two different orders of emission, summed over final states $\gamma$,

$$
\begin{aligned}
\sigma\left(\mathbf{p}_{1}, \mathbf{p}_{2}\right) \propto \sum_{\gamma} \mid & T_{\gamma i}\left(\mathbf{p}_{2}, \mathbf{p}_{1}\right) \pm\left. T_{\gamma i}\left(\mathbf{p}_{1}, \mathbf{p}_{2}\right)\right|^{2} 2 \pi \delta \\
= & \sum_{\gamma}\left[\left|T_{\gamma i}\left(\mathbf{p}_{2}, \mathbf{p}_{1}\right)\right|^{2}+\left|T_{\gamma i}\left(\mathbf{p}_{1}, \mathbf{p}_{2}\right)\right|^{2}\right] 2 \pi \delta \\
& \pm \sum_{\gamma} 2 \operatorname{Re}\left[T_{\gamma i}^{*}\left(\mathbf{p}_{2}, \mathbf{p}_{1}\right) T_{\gamma i}\left(\mathbf{p}_{1}, \mathbf{p}_{2}\right)\right] 2 \pi \delta \\
\equiv & \sigma_{\mathrm{dir}}\left(\mathbf{p}_{1}, \mathbf{p}_{2}\right) \pm \sigma_{\mathrm{int}}\left(\mathbf{p}_{1}, \mathbf{p}_{2}\right) .
\end{aligned}
$$

Here, the upper or lower sign is appropriate depending upon whether the two emitted particles are spatially symmetric or antisymmetric, and the term $2 \pi \delta$ is a shorthand notation for $2 \pi \delta\left(E-E_{\gamma}-E_{1}-E_{2}\right)$. 
The cross section is thus composed of direct and interference terms. Both of these can be evaluated with the usual assumption of maximal randomness of the reduced widths. ${ }^{6}$ That is, only pairwise correlations of the widths are nontrivial, and these are given by expressions such as

$$
\begin{aligned}
& \left\langle\gamma_{\gamma \beta}\left(\mathbf{p}_{2}\right) \gamma_{\gamma \beta^{\prime}}^{*}\left(\mathbf{p}_{1}\right)\right\rangle \equiv \delta_{\beta \beta^{\prime}}\left\langle\Gamma_{\gamma \beta}(\mathbf{p})\right\rangle_{g_{\gamma \beta}}\left(\mathbf{p}_{2}, \mathbf{p}_{1}\right), \\
& \left\langle\gamma_{\beta \alpha}\left(\mathbf{p}_{1}\right) \gamma_{\beta \alpha^{\prime}}^{*}\left(\mathbf{p}_{2}\right)\right\rangle \equiv \delta_{\alpha \alpha^{\prime}}\left\langle\Gamma_{\beta \alpha}(\mathbf{p})\right\rangle_{\beta \alpha}\left(\mathbf{p}_{1}, \mathbf{p}_{2}\right),
\end{aligned}
$$

which define the function $g$, and where $\mathbf{p}=\left(\mathbf{p}_{1}+\mathbf{p}_{2}\right) / 2$ $\approx p_{1} \approx p_{2}$ is the average momentum of the emitted particles and the partial widths are $\Gamma_{v \mu}=\gamma_{v \mu}^{2}$. The angular brackets denote averaging over the compound-nucleus states. Note that we assume randomness with respect to the discrete level indices, but cannot do so with respect to the continuous momentum label.

We can express the ratio of the interference cross section to the direct cross section for $\mathbf{p}_{1}=\mathbf{p}_{2}$ as

$$
\begin{aligned}
R\left(\mathbf{p}_{1}, \mathbf{p}_{2}\right) & \equiv \sigma_{\text {int }}\left(\mathbf{p}_{1}, \mathbf{p}_{2}\right) / \sigma_{\text {dir }}\left(\mathbf{p}_{1}, \mathbf{p}_{2}\right) \\
& =g_{\alpha \beta}\left(\mathbf{p}_{1}, \mathbf{p}_{2}\right) g_{\beta \gamma}\left(\mathbf{p}_{2}, \mathbf{p}_{1}\right) \frac{\left\langle\Gamma_{\beta}\right\rangle^{2}}{\left\langle\Gamma_{\beta}\right\rangle^{2}+\left(E_{2}-E_{1}\right)^{2}} \\
& \approx\left|g\left(\mathbf{p}_{1}, \mathbf{p}_{2}\right)\right|^{2} \frac{\langle\Gamma\rangle^{2}}{\langle\Gamma\rangle^{2}+\left(E_{2}-E_{1}\right)^{2}},
\end{aligned}
$$

where the last line follows if we assume that the properties of the successive compound nuclei are similar.

Equation (4) has a physically appealing form. The interference is governed by factors associated with the spatial and temporal extent of the compound nucleus. The spatial dependence is embodied in $g\left(\mathbf{p}_{1}, \mathbf{p}_{2}\right)$, which describes the momentum coherence of the particle emission matrix elements. For zero relative momentum, $g(\mathbf{p}, \mathbf{p})$ $=1$, and $g$ is expected to fall to zero with increasing relative momentum on a scale $\hbar / R$, where $R$ is the radius of the compound nucleus. The temporal dependence is embodied in the Lorentzian factor involving the energy difference $E_{1}-E_{2}$, the scale being set by the average compound-nucleus width, suggestive of Ericson-Brink fluctuations. ${ }^{7}$ Indeed, it is natural that the two-particle inclusive cross section and the autocorrelation function of a one-particle exclusive cross section have the same information content.

To make these ideas concrete, we consider evaporation of neutrons from a rapidly spinning compound nucleus, as might be formed in a heavy-ion collision. We neglect the spin of the neutrons and of the initial target and projectile nuclei as small compared to the other angular momenta of the problem. We concentrate on the dependence on the relative angle between the two emitted particles, because the difference in absolute momentum is mainly governed by the second factor in Eq. (4) which is trivially evaluated. We can then adopt, for the reduced widths, the expression

$\gamma_{v \mu}(\mathbf{p})=\eta_{v \mu} \sum_{l, m_{t}} i^{l} F_{l}(p) Y_{l m_{t}}(\hat{\mathbf{p}})\left(l m_{l} J_{v} M_{v} \mid J_{\mu} M_{\mu}\right)$

where $\eta_{v \mu}$ is a random variable having zero mean and unit variance, $J_{v, \mu}$ are the fragment angular momenta, $l$ is the angular momentum of the emitted neutron, $M_{v, \mu}$ and $m_{l}$ are the magnetic quantum numbers, and $F_{l}$ is a radial integral. Note that the definite parities of $v$ and $\mu$ restrict $l$ to only even or odd values.

Because of angular momentum conservation, we cannot factor the average of four reduced widths into the product of two averages of two reduced widths, as we did in Eq. (3). Rather, the angular dependence of the interference cross section is proportional to

$$
\begin{aligned}
& \left\langle\gamma_{\beta \alpha}^{*}\left(\mathbf{p}_{2}\right) \gamma_{\gamma \beta}^{*}\left(\mathbf{p}_{1}\right) \gamma_{\gamma \beta}\left(\mathbf{p}_{2}\right) \gamma_{\beta \alpha}\left(\mathbf{p}_{1}\right)\right\rangle \\
& =\sum_{l_{1}, m_{1}} \sum_{l_{2}, m_{2}} \sum_{l_{1}^{\prime}, m_{1}^{\prime} l_{2}^{\prime}, m_{2}^{\prime}} i^{l_{1}-l_{1}^{\prime}+l_{2}-l_{2}^{\prime}} F_{l_{1}}\left(p_{1}\right) F_{l_{1}^{\prime}}\left(p_{2}\right) F_{l_{2}}\left(p_{2}\right) F_{l_{2}^{\prime}}\left(p_{1}\right) Y_{l_{1} m_{1}}\left(\hat{\mathbf{p}}_{1}\right) Y_{l_{1}^{\prime} m_{1}^{\prime}}^{*}\left(\hat{\mathbf{p}}_{2}\right) Y_{l_{2} m_{2}}\left(\hat{\mathbf{p}}_{2}\right) Y_{l_{2}^{\prime} m_{2}^{\prime}}^{*}\left(\hat{\mathbf{p}}_{1}\right) \\
& \times \sum_{J_{\alpha}} T_{J_{\alpha}}\left(2 J_{\alpha}+1\right) \sum_{J_{\beta}} \rho_{J_{\beta}} \sum_{J_{\gamma}} \rho_{J_{\gamma}} \sum_{M_{\beta}, M_{\beta}^{\prime}, M_{\gamma}}\left(l_{1} m_{1} J_{\beta} M_{\beta} \mid J_{\alpha} 0\right)\left(l_{2} m_{2} J_{\gamma} M_{\gamma} \mid J_{\beta} M_{\beta}\right) \\
& \times\left(l_{1}^{\prime} m_{1}^{\prime} J_{\beta} M_{\beta}^{\prime} \mid J_{\alpha} 0\right)\left(l_{2}^{\prime} m_{2}^{\prime} J_{\gamma} M_{\gamma} \mid J_{\beta} M_{\beta}^{\prime}\right) .
\end{aligned}
$$

Here, $J_{\alpha}$ is the angular momentum of the initial compound nucleus, $T_{J_{a}}$ is the entrance-channel transmission coefficient, and $\rho_{J}$ describes the angular momentum distribution of the compound-nucleus levels. For neutrons detected with equal energies, the ratio of the interference to direct cross sections is given by

$$
\frac{\sigma_{\text {int }}}{\sigma_{\text {dir }}}=\frac{\left\langle\gamma_{\beta \alpha}^{*}\left(\mathbf{p}_{2}\right) \gamma_{\gamma \beta}^{*}\left(\mathbf{p}_{1}\right) \gamma_{\gamma \beta}\left(\mathbf{p}_{2}\right) \gamma_{\beta \alpha}\left(\mathbf{p}_{1}\right)\right\rangle}{\left\langle\gamma_{\beta \alpha}^{*}\left(\mathbf{p}_{1}\right) \gamma_{\gamma \beta}^{*}\left(\mathbf{p}_{2}\right) \gamma_{\alpha \beta}\left(\mathbf{p}_{2}\right) \gamma_{\beta \alpha}\left(\mathbf{p}_{1}\right)\right\rangle} .
$$

For our schematic calculation, we have assumed sharp-cutoff models for both $T_{J}$ and $F_{l}, T_{J}=\theta\left(J_{c}-J\right)$, and $F_{l}(p)=\theta\left(l_{c}-l\right)$, where $l_{c}$ can be identified as $p R, R$ being the radius of the compound nucleus. Hence, increasing $l_{c}$ can be identified with increasing neutron energy. We have also taken the standard form

$$
\rho_{J} \propto(2 J+1) \exp \left(-\frac{\left(J+\frac{1}{2}\right)^{2}}{2 \sigma^{2}}\right) .
$$

We consider three possible "paths" of directions for the emitted neutrons. In terms of the conventional polar angles, path $\mathrm{I}$ has $\phi_{1}=\phi_{2}=\pi / 2$ and $\theta_{1}=\pi / 2-\delta, \theta_{2}$ $=\pi / 2+\delta$. path II has $\theta_{1}=\theta_{2}=\pi / 2$, and $\phi_{1}=-\phi_{2}=\delta$, while path III has $\phi_{1}=0, \phi_{2}=\pi$, and $\theta_{1}=\theta_{2}=\delta$. Thus, $\delta$ 
is the half-opening angle of the pair in all cases. For each path, we calculate the ratio

$C\left(\mathbf{p}_{1}, \mathbf{p}_{2}\right)=\frac{\left\langle\gamma_{\beta \alpha}^{*}\left(\mathbf{p}_{2}\right) \gamma_{\gamma \beta}^{*}\left(\mathbf{p}_{1}\right) \gamma_{\gamma \beta}\left(\mathbf{p}_{2}\right) \gamma_{\beta \alpha}\left(\mathbf{p}_{1}\right)\right\rangle_{\delta}}{\left\langle\gamma_{\beta \alpha}^{*}\left(\mathbf{p}_{2}\right) \gamma_{\gamma \beta}^{*}\left(\mathbf{p}_{1}\right) \gamma_{\gamma \beta}\left(\mathbf{p}_{2}\right) \gamma_{\beta \alpha}\left(\mathbf{p}_{1}\right)\right\rangle_{\delta=0}}$,

which is experimentally more convenient than the ratio of the direct to the interference cross sections, but carries the same information content.

Typical interference patterns that result are shown in Fig. 1. All peak at $\delta=0$, with a width narrowing with increasing $l_{c}$. There are also small oscillations for larger opening angles whose amplitude diminishes as $l_{c}$ increases.

An experimental measure of the source "radius" is given by the small-angle behavior of the ratio $C$. The correlation function is usually written as

$C\left(\mathbf{p}_{1}, \mathbf{p}_{2}\right)=\int d \mathbf{r}_{1} d \mathbf{r}_{2} D\left(\mathbf{r}_{1}, \mathbf{p}\right) D\left(\mathbf{r}_{2}, \mathbf{p}\right) \cos \left[\mathbf{q} \cdot\left(\mathbf{r}-\mathbf{r}_{2}\right)\right]$,

where the source distribution $D(\mathbf{r}, \mathbf{p})$ is assumed to have unit spatial integral and where $\mathbf{q}$ is the relative momentum, whose magnitude is given by $q=2 p \sin \delta$. Expanding Eq. (10) to second order in $q$ and performing the integrations over $\mathbf{r}_{1}$ and $\mathbf{r}_{2}$ yields

$$
C\left(\mathbf{p}_{1}, \mathbf{p}_{2}\right)=1-\frac{1}{3} q^{2}\left\langle r^{2}\right\rangle,
$$

with $\left\langle r^{2}\right\rangle=\int d \mathbf{r} D(\mathbf{r}, \mathbf{p}) r^{2}$ the mean-square source radius. Thus, the second derivative of $C$ with respect to $\delta$ at

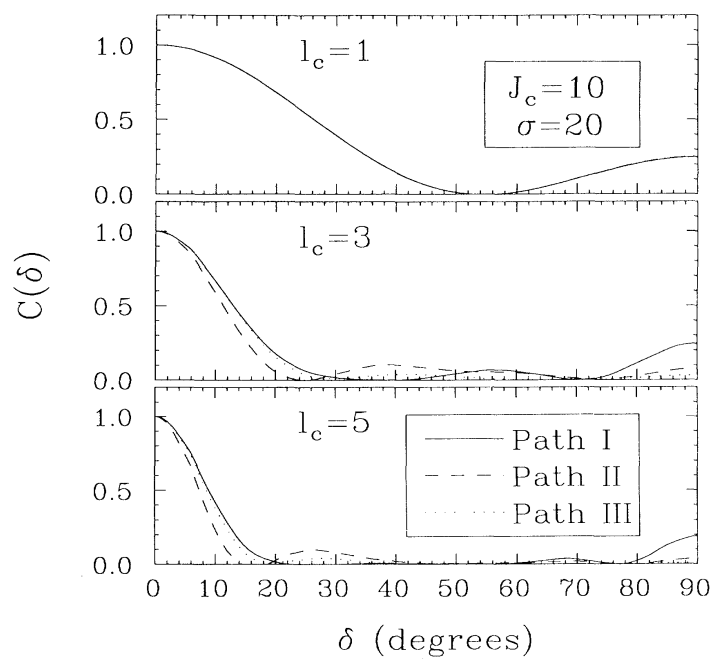

FIG. 1. Correlation functions calculated for several different values of the neutron-cutoff angular momentum $l_{c}$; the halfopening angle of the neutron pair is $\delta$. The three different lines correspond to the paths described in the text. Results are qualitatively similar for other values of the compound-nucleus parameters $J_{c}$ and $\sigma$. $\delta=0$ is given by

$\left.\frac{d^{2} C\left(\mathbf{p}_{1}, \mathbf{p}_{2}\right)}{d \delta^{2}}\right|_{\delta=0}=-\frac{8}{3} p^{2}\left\langle r^{2}\right\rangle \equiv-\frac{8}{3 \kappa} l_{c}\left(l_{c}+1\right)$,

where $\kappa$ is defined by $\left\langle r^{2}\right\rangle=R^{2} / \kappa=l_{c}\left(l_{c}+1\right) / p^{2} \kappa . \quad \kappa$ clearly depends upon the effective source geometry and has the values 1 and $\frac{5}{3}$ for surface and volume emission, respectively.

In Fig. 2, we display the effective source geometry as extracted from our correlation functions for various values of $l_{c}$. Note that $l_{c}=5$ corresponds to a neutron kinetic energy of $20 \mathrm{MeV}$ if a nuclear radius of $R=5 \mathrm{fm}$ is assumed. Whereas the source radius extracted from path II is consistent with surface emission $(\kappa=1)$, paths I and III yield smaller radii, consistent with volume emission for higher values of $l_{c}$. This behavior persists for other reasonable compound-nucleus parameters, although equal radii are found for all three paths when $J_{c}=0$.

The physical origin of this difference in effective source radii is easy to understand. The angular momentum coupling favors neutron emission in the plane perpendicular to the fragment angular momentum. The emission probability thus decreases more rapidly perpendicular to this plane (path II) than in the other plane (paths I and III), suggesting a larger radius. We therefore conclude that the source radius obtained from interferometry has to be interpreted with care. The reaction mechanism can introduce corrections and anisotropies that do not correspond to the geometry of the emitting source, and that can be of the same order of magnitude as the latter. This conclusion should be valid for all types of reactions, although our specific calculation has been for compound-nucleus processes. It should also be noted that our calculation has not taken into account the final-state interaction between the emitted neutrons, which distorts the correlation significantly. ${ }^{5}$

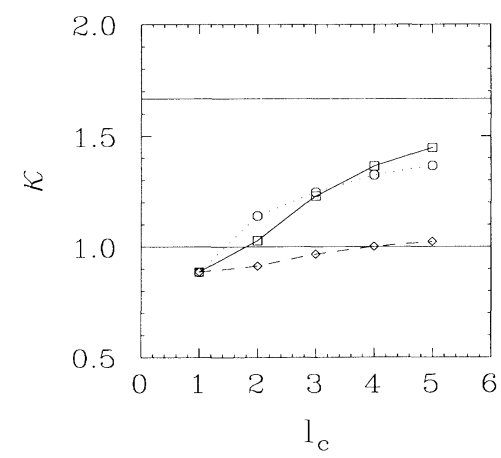

FIG. 2. Radii extracted from the small-angle correlation functions measured along the three different paths as functions of $l_{c}$. The compound-nucleus parameters are as in Fig. 1. For comparison, the horizontal lines corresponding to $\kappa=1$ and $\frac{5}{3}$ are shown. 
In conclusion, we have derived a simple formula [Eq. (4)] for quantal interference effects in the inclusive emission of two particles from a compound nucleus. The result involves the momentum and energy coherence of the partial widths in a manner similar to that describing fluctuations of exclusive cross sections. In a schematic application, we have calculated the correlation function for two neutrons emitted from a rapidly spinning compound nucleus. We found that the source size extracted from the small-angle correlation of equal-energy neutrons scaled with the nuclear radius, but showed a distinct dependence upon the direction of emission relative to the beam.

This work was supported in part by the National Science Foundation, Grants No. PHY86-04197, No. PHY85-05682, and No. PHY86-11210.
${ }^{1}$ G. F. Bertsch and S. Das Gupta, Phys. Rep. 160, 189 (1988); H. Stöcker and W. Greiner, Phys. Rep. 137, 277 (1986).

${ }^{2}$ R. Hanbury-Brown and R. Twiss, Nature (London) 178, 1046 (1956).

${ }^{3}$ C.-K. Gelbke and D. H. Boal, Prog. Part. Nucl. Phys. 19, 33 (1987); A. Bamberger et al., Phys. Lett. B 203, 320 (1988);

T. Åkesson et al., Z. Phys. C 36, 517 (1987).

${ }^{4}$ G. Bertsch, M. Gong, and M. Tohyama, Phys. Rev. C 37, 1896 (1988); S. Pratt, Phys. Rev. Lett. 53, 1219 (1984).

${ }^{5}$ S. E. Koonin, Phys. Lett. 70B, 43 (1977).

${ }^{6} \mathrm{H}$. Herman Feshbach, A. Kerman, and S. E. Koonin, Ann. Phys. (N.Y.) 125, 429 (1980).

${ }^{7}$ T. Ericson, Phys. Rev. Lett. 5, 430 (1960); D. M. Brink, et al., Nucl. Phys. 54, 577 (1964). 